\title{
Qualidade do cuidado ao acidente vascular cerebral isquêmico no SUS
}

\author{
Quality of care for ischemic stroke in the \\ Brazilian Unified National Health System
}

\author{
1 Secretaria de Saúde do \\ Distrito Federal, Brasília, \\ Brasil. \\ 2 Escola Nacional de Saúde \\ Pública Sergio Arouca, \\ Fundação Oswaldo Cruz, \\ Rio de Janeiro, Brasil. \\ Correspondência \\ C. L. R. C. Rolim \\ Secretaria de Saúde do Distrito \\ Federal. \\ SMLN, trecho 7, chácara \\ 236, Lago Norte, Brasília, DF \\ 70000-000, Brasil. \\ clrcrolim@yahoo.com.br
}

\begin{abstract}
The hospital mortality rate is an outcome measure of care used as an indicator of quality of care for stroke. Computerized tomography is the main imaging method for diagnosing and managing stroke. This study aimed to assess the quality of hospital care for acute ischemic stroke (AIS) in the Brazilian Unified National Health System (SUS), considering the severity of cases and use of computerized tomography. Multivariate analysis was used to adjust the mortality risk, evaluate the use of computerized tomography, and rank the performance of hospitals from April 2006 to December 2007. 16,879 admissions were analyzed, and at least one CT scan was performed in $28.6 \%$ of the admissions. The crude mortality rate was $34.3 \%$, and the adjusted rate was $31.2 \%$. Performance of a CT scan showed a protective effect: the adjusted OR was 0.27 for one test and 0.32 for two tests. Under-utilization of computerized tomography is one of the limiting factors for good medical practice in the management of ischemic stroke in the SUS.
\end{abstract}

Stroke; Hospital Mortality; Quality of Health Care; Tomography; Hospital Information Systems
Cristina Lúcia Rocha Cubas Rolim 1

Monica Martins 2

\section{Introdução}

O acidente vascular cerebral (AVC) é definido como uma síndrome que consiste no desenvolvimento rápido de distúrbios clínicos focais da função cerebral, global no caso do coma, que duram mais de 24 horas ou conduzem à morte sem outra causa aparente que não a de origem vascular 1 . A magnitude em termos da prevalência e incidência sinaliza sua importância epidemiológica no Brasil 2. O AVC é classificado em dois grandes grupos: $\mathrm{AVC}$ isquêmico (AVCi) e o AVC hemorrágico. $\mathrm{O}$ mais frequente, com cerca de $85 \%$ dos casos, é o AVCi, que se caracteriza pela interrupção do fluxo sanguíneo (obstrução arterial por trombos ou êmbolos) em uma determinada área do encéfalo 3,4,5. No Brasil, o AVCi representa, na população nacional, segundo diferentes estatísticas, entre $53 \%$ a $85 \%$ dos casos de AVC 6 .

A taxa de mortalidade hospitalar é uma medida do resultado do cuidado, utilizada como indicador da qualidade do cuidado para procedimentos cirúrgicos e condições médicas específicas, incluindo o AVC 5,7. A mortalidade hospitalar por AVCi até 30 dias é uma das medidas usualmente utilizadas para comparar a qualidade do cuidado, isto é, de sua efetividade 5 . O pressuposto é que melhores processos de cuidado estão relacionados à redução da mortalidade de curto prazo, o que fortalece a validade 
causal entre a medida de resultado (mortalidade hospitalar) e o processo de cuidado ${ }^{8}$. Contudo, Saposnik et al. 5 sublinharam a importância da análise do óbito aos sete dias como medida da mortalidade precoce por AVCi, considerando que as decisões clínicas cruciais são tomadas na primeira semana da admissão hospitalar. Esses autores destacam ainda que a mortalidade hospitalar por AVCi até sete dias é um parâmetro fácil de ser medido, com o mérito de homogeneizar o tempo de permanência entre unidades, pois esse geralmente excede sete dias, portanto apresenta a vantagem de eliminar um importante viés na interpretação desse indicador.

$\mathrm{O}$ cuidado na fase aguda deve ser oportuno no tempo e efetivo para impedir a morte do tecido cerebral. Para que o cuidado ao AVCi seja efetivo, é necessário um conjunto mínimo de tecnologias disponíveis no tempo correto, como a realização da tomografia computadorizada idealmente dentro de até quatro horas e meia após o início dos sintomas 9 , além de outros suportes propiciados, em geral, por unidades especializadas $1,3,10,11,12$. O uso de exames de imagem para o cuidado do AVCi é relevante para o diagnóstico diferencial, a definição e a prescrição terapêutica dos cuidados adequados. A importância da tomografia computadorizada no cuidado ao paciente com AVCi é bem documentado na literatura $10,11,12,13$.

Além do processo de cuidado adequado, diversos outros fatores influenciam o resultado do tratamento do AVCi, incluindo aqueles individuais (idade, sexo, estado socioeconômico, gravidade do AVC e comorbidades) e os relacionados ao sistema de saúde como o volume de atendimento do hospital, a existência de unidade de cuidado intensivo especializada e outras intervenções 13. Enquanto indicador da qualidade do cuidado, a mortalidade hospitalar constitui uma ferramenta preliminar de rastreamento (screening) para discriminar hospitais potencialmente com risco de estarem prestando serviços de qualidade inadequada ou inferior ao padrão esperado 14,15,16. Nessa avaliação, é imprescindível um adequado ajuste de risco para fatores clínicos e demográficos do paciente, dado que o perfil de gravidade do caso interfere sobre os resultados do cuidado 17,18 .

Amplamente utilizada em outros países, majoritariamente com base no uso de dados administrativos, o uso desse tipo de abordagem é ainda escasso na nossa realidade. O objetivo desse artigo é avaliar a qualidade do cuidado hospitalar prestado no acidente vascular cerebral agudo isquêmico (AVCi) no Sistema Único de Saúde (SUS), empregando a mortalidade hospitalar precoce, isto é, até o sétimo dia de internação, considerando o perfil de gravidade dos casos e a realização de tomografia computadorizada.

\section{Métodos}

\section{Delineamento do estudo}

O desenho do estudo é observacional, e o corte, transversal, com base em dados secundários administrativos, e voltado para avaliar a qualidade do cuidado hospitalar prestado aos pacientes na fase aguda do AVCi. Cuidado na fase aguda foi definido como aquele que é prestado durante a primeira semana de hospitalização, cujo efeito é decisivo para o prognóstico do paciente. A primeira e central dimensão da qualidade do cuidado analisada foi a efetividade, e a medida de resultado foi a mortalidade hospitalar (precoce) até o sétimo dia de permanência hospitalar. A realização ou não do exame de tomografia computadorizada foi empregada como uma medida indicativa da adequação do processo de cuidado prestado. Adequação é uma dimensão da qualidade do cuidado definida como o grau com que os cuidados estão baseados no conhecimento técnico-científico existente ${ }^{19}$. A gravidade do caso mensurada pelos atributos do paciente é um importante fator de confundimento para a avaliação do resultado do cuidado e influi também nas características do processo 7,18.

\section{Fonte de dados}

O Sistema de Informação Hospitalar (SIH) do SUS foi a principal fonte de informação utilizada. Para criar a base de dados, foram utilizados os arquivos dos tipos registro reduzido e procedimentos especiais do SIH. Os dados registrados nos arquivos de procedimentos especiais foram utilizados somente para obter informações sobre o número de tomografias computadorizadas. Na tabela de procedimentos do $\mathrm{SIH}$, os exames de tomografia computadorizada de crânio correspondiam aos seguintes códigos: 97.013.01.3 (um exame) ou 97.013.02.1 (dois exames) 20. Posteriormente, os bancos de dados foram unificados utilizando o número do formulário da Autorização para Internação Hospitalar (AIH) como variável-chave. Os meses de janeiro a março de 2006 foram excluídos por conterem erros referentes ao número da AIH nos arquivos de procedimentos especiais. O SIH-SUS, apesar de suas limitações, vem sendo utilizado como fonte de informação em estudos de avaliação de serviços de saúde e descrição da morbidade hospitalar ${ }^{21}$.

O Cadastro Nacional de Estabelecimentos de Saúde foi também utilizado para descrever a 
oferta de tomógrafos na rede hospitalar disponíveis para o SUS. Nesse cadastro, há ausência de informação sobre a existência de tomógrafo em 21 hospitais que compõem o universo de estudo.

\section{Universo de estudo}

O universo de estudo é composto pelas hospitalizações de adultos decorrentes de AVCi na fase aguda, financiadas pelo SUS, ocorridas no Brasil entre abril de 2006 e dezembro de 2007. Para a seleção dos casos de AVCi, foi utilizado somente o código I63 - infarto cerebral, da Classificação Internacional de Doenças, 10a revisão (CID-10) 22, pois esse código, mesmo quando aplicado em fontes de dados clínicos ou administrativos, aumentou a acurácia da definição diagnóstica 23,24. Outra principal justificativa para essa restrita seleção decorreu da complexidade do manuseio da CID, que pode implicar em erro de classificação do subgrupo do AVC 23,24. A segunda razão deve-se à qualidade da informação diagnóstica codificada na fonte de dados empregada 20. Finalmente, a última justificativa foi de ordem empírica; no período de estudo, $64,7 \%$ das 328.067 internações por AVC foram codificadas na categoria residual (CID-10: I64), na qual estão os casos em que não foram determinados como AVC isquêmico ou hemorrágico. Também foi encontrada uma taxa de mortalidade de $11,5 \%$ para os casos de acidentes isquêmicos transitórios AIT; G459, corroborando com a hipótese de problemas no manuseio da CID. Internações cujo tempo de permanência foi maior que sete dias e a idade foi menor que 18 anos foram excluídas. Apesar de o foco ser sobre os casos na fase aguda, é importante ressaltar a possibilidade de estarem incluídos casos de reinternações devido a complicações do AVCi, pois não é possível distinguir, no SIH-SUS, a informação referente ao primeiro evento daquela referente aos eventos de repetição ou reinternação.

\section{Análise multivariada dos dados}

A regressão logística foi utilizada para analisar a mortalidade hospitalar, cuja primeira e fundamental etapa foi ajustá-la pelos fatores de risco do pacientes (gravidade do caso). Considerando as informações disponíveis no SIH, o modelo de ajuste de risco (modelo 1) incluiu as seguintes variáveis: idade, sexo e comorbidades. A idade foi empregada como variável categórica (18-59 anos - categoria de referência; 60-79 anos e 80-99 anos). O sexo, como variável dicotômica, cuja categoria de referência foi o sexo masculino.
Quanto às comorbidades, primeiramente, foi utilizado o índice de comorbidade de Charlson modificado por Goldstein et al. 25 para aplicação em casos de AVC. O índice de comorbidade de Charlson original é composto por 19 condições clínicas e apresenta a vantagem de associar a presença de uma comorbidade a um escore que pondera o prognóstico 26,27. A principal modificação realizada por Goldstein et al. 25 foi a exclusão de duas condições clínicas do índice de comorbidade de Charlson (ICC) original por serem essas associadas à patologia em questão; as condições clínicas excluídas foram: Hemiplegia/Paraplegia e o próprio AVC. O ICC modificado foi validado como medida de gravidade em estudo sobre mortalidade hospitalar por AVC 25. Esse estudo concluiu, também, que o ICC ajustou adequadamente os modelos construídos com informações do prontuário do paciente e de grandes bases administrativas cuja informação clínica disponível é restrita. No presente estudo, foi utilizada a adaptação das condições clínicas do ICC para a CID-10 realizada por Quan et al. 28.

Comorbidades associadas a maior risco de morte em paciente com AVC, tais como diabetes e doença renal fazem parte do ICC. Comorbidades não incluídas no ICC, mas que são importantes fatores de risco, como hipertensão (CID-10: I10), dislipidemias (CID-10: E788), obesidade (CID-10: E66), carotídea (CID-10: I652), fibrilação auricular (CID-10: I48), sedentarismo (CID10: Z723), tabagismo (CID-10: Z720; X499; T652; F170; F171; F172; F173), coagulopatia (D65; D66; D67; D68; D691; D693; D696) e alcoolismo (CID10: F10; E52; T51) foram também testadas como variáveis independentes dicotômicas no modelo de ajuste de risco por sua relevância para o prognóstico do AVCi. As comorbidades registradas no banco de dados que não apresentaram significância estatística $(\mathrm{p}>0,10)$ foram excluídas do modelo final.

Por fim, considerando a limitação do sistema de informação quanto ao registro desse tipo de dado, foi ainda introduzido, no modelo, a presença simples de comorbidade (variável dicotômica). Essa última variável, apesar de inespecífica para o AVCi, agregou poder preditivo ao modelo de risco, o que justificou sua permanência no modelo de risco final.

Com base na probabilidade de morte predita pelo modelo de ajuste de risco (modelo 1), foi calculada a mortalidade hospitalar ajustada esperada. A mortalidade predita para cada hospital equivale ao somatório das probabilidades preditas para cada internação. Posteriormente, foi calculada a razão de mortalidade (observada/ predita) para comparar hospitais segundo a qualidade do seu desempenho. Os hospitais foram 
classificados em percentis segundo a razão de mortalidade, adotaram-se os pontos de cortes testados por Noronha et al. 29 , no qual o percentil 20 representa desempenho melhor que o esperado, e o percentil 80 representa um desempenho aquém do esperado. Para a classificação dos hospitais por percentil da razão de mortalidade, hospitais sem ocorrência de óbito foram excluídos, uma vez que a razão de mortalidade igual a zero não deveria ser interpretada como bom desempenho. Hipóteses como alta taxa de transferência, baixa complexidade e volume de atendimento nessas unidades e mesmo a política de admissão podem explicar a ausência de registro de óbito. A inclusão dessas unidades levaria a um erro na estimativa da mortalidade esperada. Pode se supor que esses óbitos venham a ocorrer em outras unidades hospitalares ou mesmo fora de qualquer estabelecimento de saúde. Por esse critério, foram excluídos 490 hospitais, esses representam 55,1\% dos 890 estabelecimentos que internaram pacientes com AVCi, realizaram somente 1.760 atendimentos $(10,4 \%$ do total de casos), e as transferências para outro hospital equivaleram a $4,2 \%$.

A segunda etapa da regressão logística incluiu, no modelo de base (modelo de risco), a informação sobre a realização do número de exames de tomografia computadorizada, assumindo essa variável como indicativa da adequação do processo de cuidado, para avaliar o efeito da realização desse exame sobre os resultados do cuidado. Os modelos foram avaliados quanto à capacidade discriminativa por meio da estatística C. Essa estatística mede se um modelo distingue bem indivíduos que têm resultados ruins daqueles que têm bons resultados. Representa a proporção de todos os pares de observação aleatoriamente selecionados com resultados diferentes no qual o paciente que morre tem maior probabilidade de morrer que de sobreviver. Um modelo com capacidade de discriminação perfeita classificaria todos os pacientes com probabilidades preditas de zero ou um; toda pessoa com uma probabilidade predita de um, mas ninguém com uma probabilidade predita de zero, teria o resultado de interesse. A estatística $\mathrm{C}$ varia entre 0 e 1 , maiores valores indicam melhor discriminação 18 .

\section{Resultados}

No período de estudo, ocorreram 16.879 internações, com até 7 dias de permanência, em adultos no SUS devido ao AVCi (Tabela 1). Essas internações correspondem a 73,6\% do volume total de internações por AVCi até 30 dias de per- manência ( $\mathrm{n}=22.927$ ). O número total de óbitos por AVCi foi 5.784, o que representou uma taxa de mortalidade bruta de $34,3 \%$ para o período de internação de sete dias. Os óbitos ocorridos na primeira semana de internação contabilizam $85,2 \%$ do total de óbitos por AVCi ( $\mathrm{n}=6.789$ ). A taxa de mortalidade hospitalar precoce bruta por AVCi variou entre regiões do Brasil, a Região Norte apresentou a menor taxa (13,5\%), e a Região Sudeste, a maior taxa (46\%) (Tabela 1).

A idade média foi 64,7 anos ( $\mathrm{DP}=15,7$ anos), e a mediana, igual a 66 anos. Em relação à distribuição por sexo, 53\% dos casos foram do sexo masculino. Quanto às comorbidades, em apenas $19,1 \%$ das internações, houve o registro de um segundo diagnóstico, entretanto a taxa de mortalidade hospitalar foi maior nesse grupo. Igualmente, o escore igual ou maior que 1 do Índice de Charlson, apesar de pouco frequente (4\%), apresentou uma mortalidade hospitalar maior que o dobro do grupo com escore de gravidade zero (Tabela 1).

Somente $6 \%$ das internações utilizaram a Unidade de Terapia Intensiva (UTI), e o tipo de admissão preponderante foi a emergência (88,3\%) (Tabela 1). Quanto à distribuição do tempo de permanência, $23,5 \%$ dos casos permaneceram menos de 24 horas de internação, apesar de o percentual de casos transferidos equivaler a $4,7 \%$. A maioria dos casos $(34,8 \%)$ se concentram na faixa entre 3 e 4 dias de permanência.

Hospitais públicos e privados foram, respectivamente, responsáveis por $52,9 \%$ e $47,1 \%$ das internações por AVCi até 7 dias de permanência. A diferença entre a taxa de mortalidade precoce entre os dois tipos de prestadores foi reduzida (Tabela 1). No que se refere ao tempo médio de permanência nos hospitais públicos, essa equivaleu a 3,18 dias, e, nos privados, foi 3,07 dias.

A realização de, pelo menos, um exame de tomografia computadorizada equivaleu a $28,6 \%$ das internações (Tabela 1). Vale destacar que as regiões Sudeste e Sul realizaram 91,1\% dos exames de tomografia computadorizada nas internações analisadas. A taxa de mortalidade hospitalar bruta foi $15,5 \%$ no grupo que realizou a tomografia computadorizada em contraste com a taxa de $41,8 \%$ entre os que não realizaram o exame (Tabela 1).

Das 12.056 internações cujo exame de tomografia computadorizada não foi realizado (Tabela 1), 6.499 (59,6\%) ocorreram em estabelecimentos que dispunham de tomógrafo para o SUS. A Região Nordeste apresentou a maior porcentagem de exames de tomografia computadorizada não realizados $(85,3 \%$ - 605 casos $)$ em estabelecimentos que dispunham de tomógrafo. O segundo maior percentual foi na Região Sudeste $(66,1 \%$ 
Características das internações por acidente vascular cerebral isquêmico. Brasil, 2006-2007.

\begin{tabular}{|c|c|c|c|}
\hline Características & Número de casos (\%) & Idade média (anos) & $\begin{array}{c}\text { Taxa de mortalidade } \\
\text { hospitalar (\%) }\end{array}$ \\
\hline \multicolumn{4}{|l|}{ Paciente } \\
\hline \multicolumn{4}{|l|}{ Sexo } \\
\hline Masculino & $8.949(53,0)$ & 63,2 & 36,5 \\
\hline Feminino & $7.930(47,0)$ & 66,4 & 31,7 \\
\hline \multicolumn{4}{|c|}{ Faixa etária (anos) } \\
\hline $18-49$ & $2.830(16,8)$ & - & 45,3 \\
\hline $50-59$ & $2.951(17,4)$ & - & 34,6 \\
\hline $60-69$ & $3.895(23,1)$ & - & 29,5 \\
\hline $70-79$ & $4.273(25,3)$ & - & 30,2 \\
\hline $80-89$ & $2.430(14,4)$ & - & 33,6 \\
\hline $90-99$ & $500(3,0)$ & - & 44,8 \\
\hline \multicolumn{4}{|l|}{ Comorbidade } \\
\hline Ausente & $13.658(80,9)$ & 64,7 & 29,7 \\
\hline Presente & $3.221(19,1)$ & 64,3 & 53,8 \\
\hline \multicolumn{4}{|l|}{ Índice Charlson } \\
\hline 0 & $16.199(96,0)$ & 64,6 & 32,4 \\
\hline$\geq 1$ & $680(4,0)$ & 65,3 & 78,7 \\
\hline \multicolumn{4}{|l|}{ Processo de cuidado } \\
\hline \multicolumn{4}{|c|}{ Especialidade médica } \\
\hline Cirúrgica & $3.262(19,3)$ & 55,9 & 92,0 \\
\hline Clínica & $13.604(80,7)$ & 66,8 & 20,4 \\
\hline \multicolumn{4}{|l|}{ Tipo de admissão } \\
\hline Eletiva & $1970(11,7)$ & 64,1 & 90,0 \\
\hline Urgência & $14.907(88,3)$ & 64,7 & 26,9 \\
\hline \multicolumn{4}{|c|}{ Realização de tomografia } \\
\hline $\operatorname{Sim}$ & $4.823(28,6)$ & 64,6 & 15,4 \\
\hline Não & $12.056(71,4)$ & 64,7 & 41,8 \\
\hline \multicolumn{4}{|l|}{ Uso de UTI } \\
\hline Sim & $1.013(6,0)$ & 60,8 & 32,5 \\
\hline Não & $15.866(94,0)$ & 64,9 & 61,8 \\
\hline \multicolumn{4}{|l|}{ Tipo de hospital } \\
\hline \multicolumn{4}{|l|}{ Natureza jurídica } \\
\hline Público & $8921(52,9)$ & 63,8 & 33,9 \\
\hline Privado & $7958(47,1)$ & 65,6 & 34,7 \\
\hline \multicolumn{4}{|c|}{ Distribuição geográfica } \\
\hline Norte & $326(1,9)$ & 65,4 & 13,5 \\
\hline Nordeste & $3.231(19,1)$ & 66,2 & 24,8 \\
\hline Sudeste & $8.021(47,6)$ & 64,6 & 46,0 \\
\hline Sul & $4.664(27,6)$ & 63,9 & 24,8 \\
\hline Centro-oeste & $637(3,8)$ & 63,5 & 14,1 \\
\hline Total & $16.879(100,0)$ & 64,7 & 34,3 \\
\hline
\end{tabular}

UTI: Unidade de Terapia Intensiva.

Fonte: Sistema de Informação Hospitalar, Departamento de Informática do SUS, Ministério da Saúde. 
- 4.230 casos). A Região Sul foi a região com menor porcentagem de exames não realizados em estabelecimentos com tomógrafo disponível, mesmo assim, 1.371 (41,9\%) internações por AV$\mathrm{Ci}$ foram atendidas em locais com equipamento disponível e não fizeram o exame.

A comparação entre os grupos que realizaram e aqueles que não realizaram tomografia computadorizada mostrou um efeito protetor para o risco de morrer associado à realização desse exame (odds ratio - OR $=0,25$; intervalo de 95\% de confiança - IC95\%: 0,23-0,27). As diferenças foram favoráveis à realização de tomografia computadorizada mais precocemente no tempo, pois o grupo que realizou o exame antes de $24 \mathrm{~h}$ de internação apresentou uma taxa de mortalidade hospitalar bruta de $30,7 \%$, em contraste com a taxa de mortalidade de $95,4 \%$ entre os que não realizaram o exame - $(\mathrm{p}<0,000)$, com OR de 0,02 (IC95\%: 0,01-0,03). A relação se manteve favorável ao grupo que realizou o exame com um dia de internação (OR = 0,36; IC95\%: 0,27-0,47) e com dois dias de internação (OR = 0,60; IC95\%: 0,46-0,79). No grupo que permaneceu entre $3 \mathrm{e}$ 4 dias, essa relação se inverteu, com mortalidade e OR maior para aqueles que realizaram o exame. Nos casos que permaneceram cinco ou mais dias, não foi observado esse padrão, pois o grupo que realizou a tomografia computadorizada tardiamente teve taxa de mortalidade hospitalar bruta ligeiramente menor $(12,5 \%)$ que o grupo que não realizou o exame (13,8\%), e o OR não foi significativo.

No modelo final para a predição do risco de morrer para o AVCi até o sétimo dia de internação, permaneceram estatisticamente significativas as seguintes variáveis: idade, sexo, ICC e presença de comorbidade selecionadas (Tabela 2: modelo 1). Diferentemente do índice de Charlson e da presença de comorbidades, a variável faixa etária apresentou um efeito protetor sobre a chance de morrer para os maiores de 60 anos em relação à categoria de referência ( $\leq 59$ anos). Esse modelo apresentou uma capacidade preditiva razoável (estatística C: 0,67).

A inserção da variável referente ao número de exames realizados no modelo de ajuste de risco (Tabela 2: modelo 2) melhorou a capacidade preditiva do modelo, estatística C igual a 0,72. A realização de um exame de tomografia apresentou efeito protetor, com um OR ajustado de 0,27 (IC95\% 0,25-0,30) (Tabela 2).

Com base na razão de mortalidade, os 400 hospitais classificados apresentaram, no grupo com melhor desempenho, variação na razão de mortalidade entre 0,04 e 0,44. No extremo oposto, a variação da razão de mortalidade entre hospitais com pior desempenho foi entre 2,62 e 4,20
(Tabela 3). Hospitais classificados no percentil 20 apresentaram a maior transferência de casos comparativamente aos hospitais do percentil 80, isso pode ter impactado a taxa de mortalidade hospitalar precoce bruta. Também se observou disparidade entre esses dois grupos com relação ao volume de exames de tomografia computadorizada realizados, mas essa diferença não parece se explicar pela oferta de tomógrafos (Tabela 3). Visto de outra forma, significa que, comparativamente, os hospitais classificados no percentil 20 realizaram um número de exames de tomografia computadorizada por internação $(44,7 \%$ das internações) muito superior ao número de exames por internação realizados no percentil 80 (7,1\%). Com base no número de óbitos preditos (Tabela 3), a taxa de mortalidade hospitalar precoce ajustada por risco foi 31,2\% [5.259/16.879] quando se considera o volume de internações até 7 dias, resultando num razão de mortalidade de 1,10. A taxa de mortalidade precoce ajustada considerando o total de internações decresce para 22,9\% [5.259/22.927].

\section{Discussão}

Os principais achados desse estudo mostraram que as taxas de mortalidade hospitalar precoce bruta $(34,3 \%)$ e ajustada $(31,2 \%)$ são muito elevadas quando comparadas, por exemplo, à taxa canadense de 6,9\% encontrada por Saposnik et al. 5,17 ou à taxa holandesa relatada por Ogbu et al. 30 de $17,3 \%$. As taxas no SUS diminuem um pouco se consideramos, no denominador, o volume total de internações por AVCi; a taxa bruta passa para 29,6 [5.784/22.927], e a taxa ajustada, para 25,2\% [5.259/22.927], mas permanecem altas. A realização de exame de tomografia apresentou um efeito protetor, OR ajustado de 0,27 para um exame e 0,32 para a realização de dois exames.

Apesar de a tomografia computadorizada ser indicada para uma conduta terapêutica adequada dos casos de AVC, observou-se, neste estudo, tanto o grau insuficiente de incorporação dessa tecnologia como a subutilização desse exame para os casos de AVCi internados no SUS, evidenciada pelo baixo percentual $(28,6 \%)$ da realização do exame de tomografia computadorizada. Parcialmente, isso se deve ao número de equipamentos disponíveis na rede hospitalar do SUS, apenas 30,8\% dos estabelecimentos disponibilizam tomógrafos para o SUS. Contudo, observou-se também um pequeno número de exames realizados, mesmo em hospitais com o aparelho disponível. As desigualdades regionais quanto ao acesso à tomografia computadorizada 
Modelo logístico para predição do risco de morte precoce * por acidente vascular cerebral isquêmico. Brasil, 2006-2007.

\begin{tabular}{|c|c|c|c|c|c|c|}
\hline Variáveis independentes & \multicolumn{3}{|c|}{ Modelo de risco (1) } & \multicolumn{3}{|c|}{ Modelo final (2) } \\
\hline Constante & $-0,546$ & 0,033 & - & $-0,238$ & 0,035 & \\
\hline Sexo (categoria de referência $=$ homem) & $-0,184$ & 0,035 & $0,83(0,78-0,89)$ & $-0,170$ & 0,036 & $0,84(0,79-0,91)$ \\
\hline \multicolumn{7}{|c|}{ Índice de Charlson (categoria de referência: escore $=0$ ) } \\
\hline Registro de comorbidade * & 1,731 & 0,061 & $5,65(5,01-6,36)$ & 1,645 & 0,063 & $5,18(4,58-5,86)$ \\
\hline \multicolumn{7}{|l|}{ Idade (categoria de referência: $\leq 59$ anos) } \\
\hline $60-79$ anos & $-0,434$ & 0,038 & $0,65(0,60-0,70)$ & $-0,452$ & 0,039 & $0,64(0,59-0,69)$ \\
\hline 80-99 anos & $-0,173$ & 0,050 & $0,84(0,76-0,93)$ & $-0,237$ & 0,051 & $0,79(0,71-0,87)$ \\
\hline Arritmia ** & $-1,785$ & 0,376 & $0,17(0,08-0,35)$ & $-1,211$ & 0,39 & $0,30(0,14-0,64)$ \\
\hline \multicolumn{7}{|c|}{$\begin{array}{l}\text { Realização de tomografia computadorizada (categoria } \\
\text { de referência: não) }\end{array}$} \\
\hline 1 exame & - & - & - & $-1,298$ & 0,049 & $0,27(0,25-0,30)$ \\
\hline 2 exames & - & - & - & $-1,146$ & 0,114 & $0,32(0,25-0,40)$ \\
\hline
\end{tabular}

OR: odds ratio; IC95\%: intervalo de 95\% de confiança.

Fonte: Sistema de Informação Hospitalar, Departamento de Informática do SUS, Ministério da Saúde.

* Mortalidade hospitalar precoce equivale aos óbitos ocorridos até o sétimo dia de internação;

** Categoria de referência foi a ausência do atributo.

Propriedades do modelo 1 (risco): estatística C =0,67; IC95\%: 0,66-0,68; $\chi^{2}$ modelo: 1833,84 ( $p=0,000$ ); -2 log likelihood do modelo: 19885,58 .

Propriedades do modelo 2 (tomografia computadorizada): estatística C = 0,72; IC95\%: 0,71-0,73; $\chi^{2}$ modelo: 2730,02

$(p=0,000) ;-2$ log likelihood do modelo: 18969,41.

\section{Tabela 3}

Algumas características da rede hospitalar segundo a distribuição da razão da mortalidade hospitalar por acidente vascular cerebral isquêmico. Brasil, 2006-2007.

\begin{tabular}{|c|c|c|c|c|}
\hline Características da rede hospitalar & \multicolumn{3}{|c|}{ Razão de mortalidade (Percentil) } & Total \\
\hline Número de internações (\%) & $4.697(31,1)$ & $8.891(58,8)$ & $1.531(10,1)$ & $15.119(100,0)$ \\
\hline Volume médio (variação) & $58,7(7-490)$ & $37,0(1-1.364)$ & $19,1(1-353)$ & $37,8(1-1.364)$ \\
\hline Número de óbitos (\%) & $420(7,3)$ & $3.937(68,1)$ & $1.427(24,7)$ & $5.784(100,0)$ \\
\hline Mortalidade bruta (variação) & $8,9(1-29,3)$ & $44,3(12,2-100,0)$ & $93,2(71,4-100,0)$ & $38,3(1-100,0)$ \\
\hline Mortalidade predita (variação) & $32,2(26,8-69,4)$ & $36,6(22,4-84,1)$ & $32,1(23,8-36,7)$ & $34,8(22,4-84,1)$ \\
\hline Razão de mortalidade (variação) & $0,28(0,04-0,44)$ & $1,20(0,44-2,62)$ * & $2,90(2,62-4,20)$ & $1,10(0,04-4,20)$ \\
\hline Transferências (variação) & $8,4(0-44,1)$ & $3,6(0-50,0)$ & $0,1(0-0,3)$ & $4,7(0-50,0)$ \\
\hline Número de tomógrafo (\%) & $29(15,3)$ & $117(61,6)$ & $44(23,2)$ & $190(100,0)$ \\
\hline
\end{tabular}

* Foram retirados os 490 hospitais sem ocorrência de óbito, logo, com taxa de mortalidade hospitalar e razão de mortalidade igual a zero.

Fonte: Sistema de Informação Hospitalar, Departamento de Informática do SUS, Ministério da Saúde. 
são acentuadas, mais de $90 \%$ dos exames são realizados nas regiões Sul e Sudeste. Na análise multivariada, a introdução da variável sobre a realização de exame de tomografia computadorizada no modelo de base para predição de óbito, além de melhorar a capacidade de discriminação do modelo, apresentou um efeito protetor quanto ao risco de óbito hospitalar ajustado comparativamente a não realização desse exame.

Em relação ao volume de atendimentos nos hospitais, observou-se um valor médio de 37,8 internações, o que parece evidenciar o papel preponderante de hospitais de pequeno porte nessa rede assistencial. Isso aponta a pulverização do atendimento em estabelecimentos com baixa complexidade, cuja estrutura, provavelmente, não contempla os recursos essenciais ao cuidado à doença. A baixa complexidade dessa rede é também ilustrada pelo acesso restrito dessa população à unidade de terapia intensiva (6\% das internações). Comparativamente, o uso de UTI no Canadá atinge $25 \% 17$.

A idade média (64,7 anos) dos pacientes internados com AVCi no SUS no período de estudo foi menor que as relatadas em estudos sobre mortalidade hospitalar de outros países. No estudo canadense, a idade média era de 72 anos 5 , e, de 73,1 anos ${ }^{30}$ no estudo holandês. Além da idade média mais jovem dos pacientes brasileiros, observou-se, na análise multivariada, um efeito protetor sob o risco de morrer para pacientes com idade maior que 59 anos. Esses achados levantam a preocupação quanto (i) ao adoecimento precoce da população brasileira, ao menos daquela atendida no SUS; (ii) à urgência na adoção de medidas preventivas face aos fatores de risco modificáveis; e (iii) à revisão de protocolos e ações educativas voltados para o diagnóstico correto dessa doença nos grupos populacionais teoricamente menos expostos ao evento.

\section{Limites do estudo}

Um dos principais limites do presente estudo está associado a problemas na suficiência e qualidade das informações registradas no SIH-SUS 18,27. Assim, vale lembrar que o padrão de mortalidade e uso de exames de imagem descritos podem ser também fruto da qualidade da informação registrada. As limitações na qualidade das informações, como o baixo percentual de registro de comorbidades (19,1\%), impactam a precisão de modelos para o cálculo da mortalidade hospitalar precoce ajustada por risco 27 . Embora a probabilidade seja reduzida em função do recorte do tempo de permanência utilizado, é necessário ressaltar que os dados utilizados não permitem diferenciar os casos que são primeiro episódio de AVC daqueles recidivantes. Assim, é possível que alguns pacientes que tiveram alta hospitalar e tenham voltado a usar os serviços de saúde sejam computados mais de uma vez. Isso influencia falsamente a diminuição da taxa de mortalidade hospitalar 31, uma vez que o mesmo paciente é contado mais de uma vez no denominador. Por fim, o desenho do estudo empregou somente duas medidas das dimensões do desempenho clínico, mortalidade como medida da efetividade e o uso de tomografia computadorizada como medida da adequação do processo de cuidado prestado. Assim, considerando a complexidade da avaliação da qualidade do cuidado, o uso de múltiplas medidas de várias dimensões do desempenho aportaria um quadro mais completo. Vale ainda ressaltar que não foram testadas a validade da realização de tomografia computadorizada como medida de adequação e da mortalidade hospitalar como medida de efetividade comparativamente a aspectos mais precisos da qualidade do processo de cuidado (padrão ouro). Contudo, essa última limitação é inerente aos estudos que comparam o desempenho dos serviços de saúde com base em dados administrativos.

Por outro lado, os resultados desse estudo apresentam vários pontos relevantes, a saber: a análise cobre todo o território nacional e os vários tipos de prestadores de serviço hospitalar do SUS. Sobretudo, esse estudo explora o uso da taxa de mortalidade precoce (até o sétimo dia precoce) ajustada como indicador da efetividade do cuidado e medida de resultado. Dimensiona também o impacto sobre o resultado do cuidado ao AVCi advindo da realização ou não do exame de tomografia, variável essa que pode indicar, em termos globais, que esses pacientes receberam um cuidado orientado pela boa prática.

Os resultados aqui descritos sugerem ainda a necessidade de ações consistentes para alcançar a melhoria do desempenho clínico nessa área. Além de ações preventivas sobre os fatores de risco modificáveis que possam alterar o perfil epidemiológico da doença, há que se buscar ampliar o acesso à tomografia computadorizada o mais precocemente possível, no período inicial da manifestação da doença. A literatura recomenda 1,3,9 que esse deva ser feito em tempo hábil para viabilizar, por exemplo, o uso adequado e seguro do trombolítico dentro das quatro primeiras horas e meia do início do evento. O diagnóstico diferencial de tumores cerebrais, a avaliação da necessidade de procedimentos como a craniectomia, a indicação para o uso de antiagregantes plaquetários e o acompanhamento de complicações se fazem por meio do exame de imagem. 
Ações e estratégias gerencias são necessárias para precisar onde estão os nós que explicam a carência da oferta de tomógrafo e os obstáculos no acesso ao exame de tomografia computadorizada mesmo nos grandes hospitais, considerando a questão do limite de financiamento de exames um ponto importante a ser investigado. Ações como a hierarquização dos atendimentos, com definições claras de quais hospitais estariam aptos a atender pacientes com AVCi agudo, devem ser amplamente divulgadas à população e fazer parte do plano de encaminhamento dos resgates pelas equipes do Serviço de Atendimento Móvel de Urgência (SAMU). O emprego rotineiro e amplo de indicadores de desempenho, tais como a mortalidade hospitalar ajustada e a razão de mortalidade, aqui empregados, é fundamental para o monitoramento da qualidade do cuidado prestado no âmbito do SUS, como, aliás, ocorre em outros países 16. Hospitais com desempenho abaixo do esperado, em especial, deveriam ser avaliados para esclarecer os motivos envolvidos e identificar possíveis soluções a serem viabilizadas. Certamente, esse monitoramento requer informações sistematizadas e de boa qualidade. No Brasil, os dados coletados e disponibilizados pelo SIH do SUS, cuja principal finalidade é o reembolso dos serviços prestados, são a única fonte de informação acessível sobre a produção hospitalar nacional financiada pelo governo. Portanto, esse precisa ser igualmente aprimorado, não existe uma política que incentive o registro completo e correto da informação 27.

\section{Resumo}

A taxa de mortalidade hospitalar é uma medida do resultado do cuidado, utilizada como indicador da qualidade do cuidado para o acidente vascular cerebral (AVC). A tomografia computadorizada é o principal método de imagem utilizado para o diagnóstico e condução terapêutica dessa patologia. O objetivo deste artigo é avaliar a qualidade do cuidado hospitalar ao AVC isquêmico (AVCi) agudo no SUS, considerando o perfil de gravidade dos casos e a realização de tomografia computadorizada. Análise multivariada foi empregada para ajustar a mortalidade por risco, avaliar o uso de tomografia e classificar o desempenho dos hospitais no período entre abril de 2006 e dezembro de 2007. 16.879 internações foram analisadas. A realiza-
Em síntese, o uso da mortalidade hospitalar precoce por AVCi e a compreensão dos fatores envolvidos podem trazer insumos importantes para a melhoria da qualidade do cuidado hospitalar. Contudo, outros estudos são necessários para conhecer, com maior detalhe, o processo de cuidado hospitalar prestado ao paciente com AVC. A realização de tomografia computadorizada influiu como esperado sobre a diminuição do risco de óbito hospitalar por AVCi no SUS, principalmente quando realizada nos dois primeiros dias de internação. No SUS, a subutilização dessa tecnologia é expressiva, seja devido à oferta, ao acesso ou ao baixo emprego dessa na patologia em questão. Nesse sentido, o caminho parece ser longo, dados divulgados pelo Instituto Brasileiro de Geografia e Estatística (IBGE) em novembro de 2010 (Estatísticas da Saúde: Assistência Médico-Sanitária 2009 - http://www.ibge.gov.br/home/estatistica/popu lacao/condicaodevida/ams/2009, acessado em 09/Fev/2011) descrevem uma oferta de tomógrafo na rede SUS muito inferior à disponível para usuários dos planos privados da saúde suplementar ou mesmo em países como Canadá ou Portugal. Contudo, a oferta de equipamento e barreiras de acesso precisam ser suplantadas para que pacientes do SUS possam se beneficiar dessa tecnologia quando seu uso é recomendado $32,33,34$. ção de, pelo menos, um exame de tomografia computadorizada equivaleu a $28,6 \%$ das internações. A taxa de mortalidade bruta foi 34,3\%, e a taxa ajustada, $31,2 \%$. A realização de exame de tomografia computadorizada apresentou um efeito protetor: OR ajustado de 0,27 para um exame e de 0,32 para a realização de dois exames. A subutilização da tomografia computadorizada é um dos fatores limitantes para a boa prática médica no tratamento do AVCi no SUS.

Acidente Cerebral Vascular; Mortalidade Hospitalar; Qualidade da Assistência à Saúde; Tomografia; Sistemas de Informação Hospitalar 


\section{Colaboradores}

C. L. R. C. Rolim foi responsável pela concepção, revisão da literatura, análise dos dados e elaboração escrita do manuscrito. M. Martins foi responsável pela concepção e elaboração escrita do manuscrito.

\section{Referências}

1. National Institute for Health and Clinical Excellence. Stroke: diagnosis and initial management of acute stroke and transient ischaemic attack (TIA). http://www.nice.org.uk/nicemedia/pdf/ StrokeAcuteTIAClinicalGuidelineFullVersionCon sultation.pdf (acessado em 25/Mai/2008).

2. Lotufo PA. Stroke: a neglected disease in Brazil. São Paulo Med J 2005; 123:3-4.

3. The European Stroke Organization. Recomendações para o tratamento do AVC isquêmico. http:// www.eso-stroke.org/pdf/ESO08_Guidelines_Por tuguese.pdf (acessado em 20/Mai/2008).

4. Goldman L, Ausiello D. Approach to cerebrovascular diseases. In: Goldman: Cecil medicine. 23rd Ed. Phyladelphia: Saunders; 2007. p. 2701-08.

5. Saposnik G, Hill M, O’Donnell M, Fang J, Hachinski V, Kapral MK. Variables associated with 7-day, 30-day, and 1-year; fatality after ischemic stroke. Stroke 2008; 39:2318-24.

6. Pires SL, Gagliardi RJ, Gorzoni ML. Estudo das frequências dos principais fatores de risco para acidente vascular cerebral isquêmico em idosos. Arq Neuropsiquiatr 2004; 62(3B):844-51.

7. Department of Health and Human Services and Agency for Healthcare Research and Quality. Guide to inpatient quality indicators: quality of care in hospitals. http://www.fdhc.state.fl.us/SCHS/pdf/ iqi_guide_rev4.pdf (acessado em 12/Mar/2008).
8. Donabedian A. Explorations in quality assessment and monitoring. The criteria and standards of quality. Ann Arbor: Health Administration Press; 1982.

9. Lansberg MG, Bluhmki E, Thijs VN. Efficacy and safety of tissue plasminogen activator 3 to 4.5 hours after acute ischemic stroke: a metaanalysis. Stroke 2009; 40:2438-41.

10. Hospital Israelita Albert Einstein. Protocolos gerenciados. http://www.rcplondon.ac.uk/pubs/ books/stroke/stroke_guidelines_2ed.pdf (acessado em 16/Nov/2008).

11. Wardlaw JM, Keir SL, Seymour J, Lewis S, Sandercock PAG, Dennis MS. What is the best imaging strategy for acute stroke? Health Technol Assess 2004; 8(1):iii, ix-x, 1-180.

12. Gagliardi RJ, Raffin CN, Fábio SRC. Tratamento da fase aguda do acidente vascular cerebral; Projeto Diretrizes. http://www.projetodiretrizes.org. br/projeto_diretrizes/010.pdf (acessado em 25/ Mai/2008).

13. Saposnik G, Baibergenova A, O'Donnell M, Hill MD, Kapral MK, Hachinski V, et. al. Hospital volume and stroke. Does it matter? Neurology 2007; 69:1142-51.

14. Travassos C, Noronha JC, Martins M. Mortalidade hospitalar como indicador de qualidade: uma revisão. Ciênc Saúde Coletiva 1999; 4:367-81. 
15. Robinowitz DL, Dudley A. Public reporting of provider performance: can its impact be made greater? Annu Rev Public Health 2006; 27:517-36.

16. Fung CH, Lim YW, Mattke S, Damberg C, Shekelle PG. Systematic review: the evidence that publishing patient care performance data improves quality of care. Ann Intern Med 2008; 148:111-23.

17. Saposnik G, O’Donnell M, Fang J, Hachinski V, Kapral MK, Hill M. Escalating levels of access to in-hospital care and stroke mortality. Stroke 2008; 39:2522-30.

18. Iezzoni L. Risk adjustment for measuring health care outcomes. $3^{\text {rd }}$ Ed. Ann Arbor: Health Administration Press; 2003.

19. Viacava F, Almeida C, Caetano R, Fausto M, Macinko J, Martins M, et al. Uma metodologia de avaliação do desempenho do sistema de saúde brasileiro. Ciênc Saúde Coletiva 2004; 9:711-24.

20. Secretaria de Atenção à Saúde, Ministério da Saúde. Manual do Sistema de Informações Hospitalares do SUS (SIH/SUS), 2005. http://dtr2001.saude.gov. $\mathrm{br} / \mathrm{sas} /$ download/MANUAL\%20DO\%20SIH-\%20 DEZEMBRO\%20DE\%202005\%20-\%20VERSAO\%20 FINAL-b.pdf (acessado em 10/Out/2008).

21. Bittencourt SA, Camacho LAB, Leal, MC. O Sistema de informação hospitalar e sua aplicação na saúde coletiva. Cad Saúde Pública 2006; 22:19-30.

22. Organização Mundial da Saúde. Classificação Estatística Internacional de Doenças e Problemas Relacionados à Saúde - 10a Revisão. 2a Ed. São Paulo: Editora da Universidade de São Paulo; 1995.

23. Benesch C, Witter DMJ, Wilder AL, Duncan PW, Samsa GP, Matchar DB. Inaccuracy of the International Classification of Diseases (ICD-9-CM) in identifying the diagnosis of ischemic cerebrovascular disease. Neurology 1997; 49:660-4.

24. Goldstein LB. Accuracy of ICD-9-CM coding for the identification of patients with acute ischemic stroke: effect of modifier codes. Stroke 1998; 29:1602-4.

25. Goldstain LB, Samsa GP, Matchar DB, Horner RD. Charlson index comorbidity adjustment for ischemic stroke outcome studies. Stroke 2004; 35:1941-5.
26. Martins M, Travassos C, Noronha JC. Sistema de Informações Hospitalares como ajuste de risco em índices de desempenho. Rev Saúde Pública 2001; 35:185-92.

27. Martins M. Uso de medidas de comorbidades para predição de risco de óbito em pacientes brasileiros hospitalizados. Rev Saúde Pública 2010; 44:448-56.

28. Quan H, Sundarajan V, Halfon P, Fong A, Burnand $\mathrm{B}$, Luthi J, et al. Coding algoritms for defining comorbities in ICD-9-CM and ICD-10 administrative data. Med Care 2005; 43:1130-9.

29. Noronha JC, Travassos C, Martins M, Campos MR, Maia P, Panezzuti R. Avaliação da relação entre volume de procedimentos e a qualidade do cuidado: o caso de cirurgia coronariana no Brasil. Cad Saúde Pública 2003; 19:1781-9.

30. Ogbu UC, Slobbe LCJ, Arah OA, Bruin A, Stronks K, Westert GP. Hospital stroke volume and casefatality revisited. Med Care 2010; 48:149-56.

31. Moon L, Moïse P, Jacobzone S. Stroke care in OECD Countries: a comparison of treatment, costs and outcomes in 17 countries. OECD Health Working Papers, $\mathrm{n}^{\circ}$. 5. http://www.sourceoecd.org/10.1787/ 380362605045 (acessado em 04/Out/2008).

32. Saposnik G, Jeerakathil T, Selchen D, Baibergenova A, Hachinski V, Kapral MK; Stroke Outcome Research Canada Working Group. Socioeconomic status, hospital volume, and stroke fatality in Canada. Stroke 2008; 39:3360-6.

33. Schwamm LH, Reeves MJ, Pan W, Smith EE, Frankel MR, Olson D, et al. Race/ethnicity, quality of care, and Outcomes in ischemic stroke. Circulation 2010; 121:1492-501.

34. Ovbiagele B. Nationwide trends in-hospital mortality among patients with stroke. Stroke 2010; 41:1748-54.

Recebido em 15/Fev/2011

Versão final reapresentada em 09/Jul/2011

Aprovado em 19/Ago/2011 